\title{
A modified harmonic oscillator approximation scheme for the dielectric constants of $\mathrm{Al}_{x} \mathrm{Ga}_{1-x} \mathrm{As}$
}

\author{
Fred L: Terry, Jr. \\ Center for High Frequency Microelectronics, Solid State Electronics Laboratory, Department \\ of Electrical Engineering and Computer Science, University of.Michigan, Ann Arbor, Michigan 48109-2122
}

(Received 22 October 1990; accepted for publication 25 February 1991)

\begin{abstract}
The dielectric functions of $\mathrm{Al}_{x} \mathrm{Ga}_{1}{ }_{-}$As have recently been measured for several $\mathrm{Al}$ mole fractions over the 1.5-6.0 eV wavelength range [D.E. Aspnes, S. M. Kelso, R. A.

Logan, and R. Bhat, J. Appl. Phys. 60, 754 (1986)]. To make use of this data to perform optical modeling for spectroscopic ellipsometry analysis of $\mathrm{Al}_{x} \mathrm{Ga}_{1-x}$ As-containing

samples, and for other optical modeling purposes, a reasonable interpolation scheme is required to estimate the dielectric functions of intermediate compounds. In this work, we will present a modified version of the harmonic oscillator approximation (HOA) of Erman et al. [M. Erman, J. B. Theeten, P. Chambon, S. M. Kelso, and D. E. Aspnes, J. Appl. Phys. 56, 2664 (1984)] to model the experimental data and interpolate between the known compositions over the $1.5-5.0 \mathrm{eV}$ range. Our model uses additional harmonic oscillators and allows each oscillator to have an independent phase. These modifications significantly improve the accuracy of the approximation for photon energies at and below the fundamental band-gap energy. This allows much more accurate modeling of refiection problems for multilayer GaAs/AlGaAs structures. We will present test of this approach with simulations of spectroscopic ellipsometry data using known data, and with measured spectroscopic ellipsometer data on $\mathrm{Al}_{x} \mathrm{Ga}_{1}$ - ${ }_{x}$ As-containing samples grown by molecular-beam epitaxy and organometallic chemical vapor deposition.
\end{abstract}

\section{INTRODUCTION}

Spectroscopic ellipsometry has been demonstrated to be a very useful technique for nondestructively determining the thicknesses, alloy compositions, and interface abruptness and smoothness of epitaxial III-V compound semiconductor device structures. ${ }^{1-4}$ However, in order to determine the alloy composition of a given material in a structure, the complex dielectric function of that material must be known as a function of the alloy composition, and there must be a numerically efficient means of interpolating the dielectric function between experimentally known alloy compositions. Recently, Aspnes et al. have published a set of dielectric constants vs wavelength for $\mathrm{Al}_{x} \mathrm{Ga}_{1}-x$ As covering nine $\mathrm{Al}$ mole fractions from 0 to $0.804 .^{5}$ Erman et al. ${ }^{6}$ have previously demonstrated that the dielectric response of single-crystal GaAs and ion-damaged GaAs can be accurately approximated for photon energies above the fundamental band edge by a series of seven harmonic oscillators. These authors have also suggested that this scheme is useful for interpolation of the dielectric constants of $\mathrm{Al}_{x} \mathrm{Ga}_{1}-x$ As. ${ }^{1}$ Aspnes et al. also suggest Erman's harmonic oscillator be applied to their data for modeling purposes. ${ }^{5}$ In this paper, we will present two sets of harmonic oscillator fits to Aspnes' data covering the $1.5-5.0 \mathrm{eV}$ photon energy range. The first uses seven harmonic oscillators and matches closely with the GaAs fit reported by Erman. ${ }^{6}$ The second set uses nine harmonic oscillators with independent phases to improve the quality of the fit in the near-infrared region. We will present demonstrations of the usefulness of these approximations using fits to both simulations using Aspnes' data, and to experimental spectroscopic ellipsometer data on molecular-beam epitaxy
(MBE) and organometallic chemical vapor deposition (MOCVD) grown GaAs/AlGaAs structures.

It should be emphasized that the goal of this work was to develop a mathematically simple, highly accurate, empirical set of approximations for the dielectric response of the $\mathrm{Al}_{x} \mathrm{Ga}_{1} \ldots \mathrm{A}$ As system. We did not attempt to directly relate the mathematical forms and constants in our model with parameters from band structure calculations for this materials system (although clearly some energies will be closely related to critical points). Very good estimates have been obtained for this system using the more physically based scheme of adding approximations for the behavior of the dielectric response around critical point energies; ${ }^{7,8}$ however, these approximations are not of sufficient numerical accuracy over the entire spectral range needed for spectroscopic ellipsometry analysis.

\section{EXPERIMENT}

Experimental data for this work was collected using a commercial spectroscopic ellipsometer, a Rudolph Research S2000, which spans the $1.5-5.0 \mathrm{eV}$ photon energy range. The system uses a $75-\mathrm{W}$. xenon lamp-collimatorfixed polarizer-rotating polarizer-sample-fixed analyzermonochromator design. The instrument derives the ellipsometric parameters $\tan \psi$ and $\cos \Delta$ from Fourier transform extractions of the second and fourth harmonics of the photomultiplier signal versus rotating analyzer position. This scheme makes the measurement relatively insensitive to ambient light, the absolute intensity of the signal, and polarization dependencies of the detector. All measurements were taken with accuracy constraints set so that the standard deviations of $\tan \psi$ and $\cos \Delta$ were both 
less than 0.005 . This accuracy constraint was violated in some cases for photon energies above about $4.6 \mathrm{eV}$, where a combination of low sample reflectivity and low intensity from the lamp required high voltages on the photomultiplier and reduced signal-to-noise ratios to the point that standard deviations of about 0.02 were the best practically available.

GaAs/AlGaAs epitaxial structures were grown by either MOCVD by Kopin Corp. of Taunton, MA, or by conventional solid source MBE by Quantum Epitaxial Devices of Bethlehem, PA.

\section{MODELS}

The dielectric constants of Aspnes et al. ${ }^{5}$ were fitted using a Levenberg-Marquardt least-squares nonlinear regression ${ }^{10}$ scheme to a series of harmonic oscillators:

$$
\epsilon=\sum_{\alpha=1}^{n} A_{\alpha}\left(\frac{1}{E+E_{\alpha}+i \Gamma_{\alpha}}-\frac{1}{E-E_{\alpha}+i \Gamma_{\alpha}}\right),
$$

where $\alpha$ is the index for a particular oscillator, $A_{\alpha}$ is the amplitude, $E$ is the photon energy, $E_{\alpha}$ is the center energy of the oscillator, and $\Gamma_{\alpha}$ is the oscillator damping coefficient. In both Tables I and II, ${ }^{11}$ where we present the parameters resulting from these fits, we have given the raw results of the fitting procedure to six digits. Clearly not all of these are significant figures; but we have chosen to present the numerical values actually used in our data analysis procedures without further editing.

Initially, the data were fitted using the seven harmonic oscillator approach of Erman et al. ${ }^{3,6}$ which assumes that all oscillators are in phase (all $A_{\alpha}$ 's are real and positive). As these authors noted, this fitting procedure does not yield good results below the fundamental band edge; therefore, these fits were performed over the energy range of $E_{0}-5.0 \mathrm{eV}$. $E_{0}$ was calculated for each alloy composition using Formula (1) in Aspnes et al..$^{5}$ The fits were made over the 1.5 (or higher) to $5.0 \mathrm{eV}$ photon energy range because: (i) this covers the range of our instrument and several other currently available systems; (ii) the 5.0-6.0 $\mathrm{eV}$ range is not important in most cases for material identification; and (iii) this range can be approximated accurately with relatively few oscillators. The results of these fits are found in Table I. An example of this fit for $\mathrm{Al}_{0.315} \mathrm{Ga}_{0.685} \mathrm{As}$ is shown in Fig. 1. We have selected this composition for our example plots because it is approximately the composition used in many MODFET structures, and because it proved to be one of the most difficult to fit accurately using our modified scheme discussed below. As expected, the fit is very good above $E_{0}$, but overestimates both $\epsilon_{1}$ and $\epsilon_{2}$ for energies below $E_{0}$. Also, there is a small but numerically significant error for energies just above $E_{0}$. These errors, while small on $\epsilon_{1}$ and $\epsilon_{2}$ vs energy plots, can be serious for parametric analysis of spectroscopic ellipsometry data for multilayer structures, and for other reflection modeling problems.

To improve the quality of the harmonic oscillator estimates, a modified harmonic oscillator scheme was adopted. First, extra oscillators were added in the lower energies. This, however, did not dramatically improve the fit with a reasonable oscillator count, primarily because of the very sharp change in both $\epsilon_{1}$ and $\epsilon_{2}$ around the fundamental band-gap energy. To improve the fit, the oscillator amplitudes were allowed to be complex numbers $\left(A_{\alpha}\right.$ $\left.=\left|A_{\alpha}\right| e_{\alpha}^{i \phi}\right)$. That is, the oscillators were allowed to have independent phases $\phi_{\alpha}$. During the regression for the oscillator parameters, the results of the summation were used without alteration. This allowed $\epsilon_{2}$ to become negative for some photon energies, but the regression procedure adjusted oscillator parameters to minimize any negative values. When the resulting set of oscillator parameters was used in fitting to measured data, the summation for $\epsilon_{2}$ was set to zero for any negative results. The value for $\epsilon_{1}$ from the summation was used without modification. This procedure results in a highly accurate approximation to the measured data and assures near Kramers-Kronig consistency over the entire range of the approximation. It was found that nine harmonic oscillators would accurately fit the experimental data. We began these fits with the values from the seven oscillator model with two additional oscillators which were closely spaced in energy (near $E_{0}$ ) and with one of these oscillators $180^{\circ}$ out of phase. This additional pair of oscillators was used to estimate the sharp features around $E_{0}$. Regression analysis was then performed with all nine oscillators' phases being allowed to vary, and high-quality fits were obtained. The parameters from these fits are summarized in Table II, and the improved quality of the fit for the Al mole fraction case of 0.315 is illustrated in Fig. 2. The quality of the fit is very good, with some slight inaccuracy still remaining around $E_{0}$. The average mean square error for the fits is $<0.03$ for all cases.

When using the harmonic oscillator models to interpolate between the experimental data for $\epsilon_{1}$ and $\epsilon_{2}$ for a given Al mole fraction $x$ we used simple linear interpolation for all oscillator parameters. While this may not be ideal, we did not have an experimental technique available to justify higher-order interpolation procedures. As can be seen by examining the tabulated values, the damping coefficient, amplitude, and phase of a given oscillator changes relatively slowly with composition, so second-order errors in these parameters are expected to be small. A higher-order interpolation model might be appropriate for the center energies, but thanks to the relatively fine increments in Aspnes' data, we again expect relatively small errors. This procedure produces smooth variation of the interpolated curves between the known data points.

We investigated a second approach, cubic polynomial parametrization of the complex oscillator coefficients, to the problem of interpolation of oscillator parameters. Since the variation with energy of some of the critical point energies of $\mathrm{Al}_{x} \mathrm{Ga}_{1}{ }_{x} \mathrm{As}$ are well described by cubic polynomials in the $\mathrm{Al}$ mole fraction, ${ }^{5}$ we repeated our fitting procedures assuming that all four oscillator parameters could be obtained from cubic functions of $x$. For example, the energy of oscillator number 3 at a given $\mathrm{Al}$ mole fraction $x$ would be given by 
TABLE I. Oscillator parameters (center energy, damping coefficient, and amplitude) for the seven oscillator model covering the $E_{0}$ to 5.0 eV photon range.

Al

Fraction $\alpha \quad E_{\alpha}(e V) \quad \Gamma_{\alpha}(e V)$
Al

Fraction $\quad \alpha \quad E_{\alpha}(\mathrm{eV}) \quad \Gamma_{\alpha}(\mathrm{eV}) \quad \mathrm{A}_{\alpha}$

$A_{\alpha} \quad$ Fraction $\quad \alpha \quad E_{\alpha}(e V) \quad \Gamma_{\alpha}(e V) \quad A_{\alpha}$

\begin{tabular}{|c|c|c|c|c|c|c|c|c|c|}
\hline 0.000 & $\begin{array}{l}1 \\
2 \\
3 \\
4 \\
5 \\
6 \\
7\end{array}$ & $\begin{array}{l}2.921020 \\
3.124340 \\
3.364390 \\
3.930110 \\
4.499710 \\
4.853060 \\
7.144760\end{array}$ & $\begin{array}{l}0.090531 \\
0.156487 \\
0.475297 \\
0.479202 \\
0.326958 \\
0.287297 \\
0.247217\end{array}$ & $\begin{array}{l}0.808077 \\
1.289900 \\
4.201350 \\
2.374380 \\
4.075830 \\
4.789250 \\
6.062710\end{array}$ & 0.491 & $\begin{array}{l}1 \\
2 \\
3 \\
4 \\
5 \\
6 \\
7\end{array}$ & $\begin{array}{l}3.288300 \\
3.523450 \\
3.838640 \\
4.152750 \\
4.702530 \\
4.705230 \\
6.377990\end{array}$ & $\begin{array}{l}0.215060 \\
0.226921 \\
0.262302 \\
0.255662 \\
0.367441 \\
0.377187 \\
0.265810\end{array}$ & $\begin{array}{l}2.005330 \\
1.640230 \\
1.369970 \\
0.988639 \\
3.256040 \\
5.869920 \\
5.612610\end{array}$ \\
\hline 0.099 & $\begin{array}{l}1 \\
2 \\
3 \\
4 \\
5 \\
6 \\
7\end{array}$ & $\begin{array}{l}2.990230 \\
3.183850 \\
3.609590 \\
4.044730 \\
4.519580 \\
4.820990 \\
7.137610\end{array}$ & $\begin{array}{l}0.074329 \\
0.272113 \\
0.362330 \\
0.402397 \\
0.305046 \\
0.320226 \\
0.265435\end{array}$ & $\begin{array}{l}0.431772 \\
3.598290 \\
2.034220 \\
2.040680 \\
3.265620 \\
5.469840 \\
6.327450\end{array}$ & 0.590 & $\begin{array}{l}1 \\
2 \\
3 \\
4 \\
5 \\
6 \\
7\end{array}$ & $\begin{array}{l}3.366990 \\
3.605980 \\
3.897130 \\
4.178930 \\
4.706490 \\
4.706490 \\
6.236730\end{array}$ & $\begin{array}{l}0.232680 \\
0.213191 \\
0.239184 \\
0.218641 \\
0.365906 \\
0.365906 \\
0.232119\end{array}$ & $\begin{array}{l}2.236480 \\
1.345070 \\
1.231280 \\
0.776088 \\
3.294060 \\
5.869920 \\
5.450760\end{array}$ \\
\hline 0.198 & $\begin{array}{l}1 \\
2 \\
3 \\
4 \\
5 \\
6 \\
7\end{array}$ & $\begin{array}{l}3.056240 \\
3.249760 \\
3.671280 \\
4.072330 \\
4.564490 \\
4.803120 \\
6.841540\end{array}$ & $\begin{array}{l}0.084201 \\
0.280080 \\
0.330901 \\
0.365187 \\
0.333281 \\
0.356770 \\
0.017379\end{array}$ & $\begin{array}{l}0.433911 \\
3.620430 \\
1.736750 \\
1.710470 \\
3.447300 \\
5.812080 \\
5.475890\end{array}$ & 0.700 & $\begin{array}{l}1 \\
2 \\
3 \\
4 \\
5 \\
6 \\
7\end{array}$ & $\begin{array}{l}3.466160 \\
3.695340 \\
3.951350 \\
4.197670 \\
4.703400 \\
4.703400 \\
6.057890\end{array}$ & $\begin{array}{l}0.231875 \\
0.199884 \\
0.199637 \\
0.195274 \\
0.369344 \\
0.369344 \\
0.221071\end{array}$ & $\begin{array}{l}1.992050 \\
1.357960 \\
1.008280 \\
0.740145 \\
3.463190 \\
5.869920 \\
5.047800\end{array}$ \\
\hline 0.315 & $\begin{array}{l}1 \\
2 \\
3 \\
4 \\
5 \\
6 \\
7\end{array}$ & $\begin{array}{l}3.121470 \\
3.315060 \\
3.725290 \\
4.110960 \\
4.649450 \\
4.765640 \\
6.837320\end{array}$ & $\begin{array}{l}0.105011 \\
0.284593 \\
0.322190 \\
0.356399 \\
0.337211 \\
0.409381 \\
0.025715\end{array}$ & $\begin{array}{l}0.519449 \\
3.451430 \\
1.568600 \\
1.524940 \\
3.564840 \\
5.869920 \\
5.330970\end{array}$ & 0.804 & $\begin{array}{l}1 \\
2 \\
3 \\
4 \\
5 \\
6 \\
7\end{array}$ & $\begin{array}{l}3.579240 \\
3.785860 \\
3.997220 \\
4.212350 \\
4.708380 \\
4.708380 \\
6.082540\end{array}$ & $\begin{array}{l}0.229747 \\
0.180598 \\
0.176558 \\
0.173953 \\
0.390873 \\
0.390873 \\
0.156314\end{array}$ & $\begin{array}{l}1.653770 \\
1.254100 \\
0.909090 \\
0.625824 \\
3.898090 \\
5.869920 \\
4.873670\end{array}$ \\
\hline 0.419 & $\begin{array}{l}1 \\
2 \\
3 \\
4 \\
5 \\
6 \\
7\end{array}$ & $\begin{array}{l}3.218530 \\
3.443350 \\
3.788520 \\
4.129240 \\
4.705230 \\
4.705230 \\
6.577580\end{array}$ & $\begin{array}{l}0.186985 \\
0.244430 \\
0.290906 \\
0.282572 \\
0.377187 \\
0.377187 \\
0.202936\end{array}$ & $\begin{array}{l}1.516500 \\
2.126240 \\
1.546290 \\
1.081240 \\
3.532650 \\
5.869920 \\
5.625540\end{array}$ & & & & & \\
\hline
\end{tabular}

\begin{tabular}{|c|c|c|c|c|c|c|c|c|c|}
\hline 0.000 & $\begin{array}{l}1 \\
2 \\
3 \\
4 \\
5 \\
6 \\
7\end{array}$ & $\begin{array}{l}2.921020 \\
3.124340 \\
3.364390 \\
3.930110 \\
4.499710 \\
4.853060 \\
7.144760\end{array}$ & $\begin{array}{l}0.090531 \\
0.156487 \\
0.475297 \\
0.479202 \\
0.326958 \\
0.287297 \\
0.247217\end{array}$ & $\begin{array}{l}0.808077 \\
1.289900 \\
4.201350 \\
2.374380 \\
4.075830 \\
4.789250 \\
6.062710\end{array}$ & 0.491 & $\begin{array}{l}1 \\
2 \\
3 \\
4 \\
5 \\
6 \\
7\end{array}$ & $\begin{array}{l}3.288300 \\
3.523450 \\
3.838640 \\
4.152750 \\
4.702530 \\
4.705230 \\
6.377990\end{array}$ & $\begin{array}{l}0.215060 \\
0.226921 \\
0.262302 \\
0.255662 \\
0.367441 \\
0.377187 \\
0.265810\end{array}$ & $\begin{array}{l}2.005330 \\
1.640230 \\
1.369970 \\
0.988639 \\
3.256040 \\
5.869920 \\
5.612610\end{array}$ \\
\hline 0.099 & $\begin{array}{l}1 \\
2 \\
3 \\
4 \\
5 \\
6 \\
7\end{array}$ & $\begin{array}{l}2.990230 \\
3.183850 \\
3.609590 \\
4.044730 \\
4.519580 \\
4.820990 \\
7.137610\end{array}$ & $\begin{array}{l}0.074329 \\
0.272113 \\
0.362330 \\
0.402397 \\
0.305046 \\
0.320226 \\
0.265435\end{array}$ & $\begin{array}{l}0.431772 \\
3.598290 \\
2.034220 \\
2.040680 \\
3.265620 \\
5.469840 \\
6.327450\end{array}$ & 0.590 & $\begin{array}{l}1 \\
2 \\
3 \\
4 \\
5 \\
6 \\
7\end{array}$ & $\begin{array}{l}3.366990 \\
3.605980 \\
3.897130 \\
4.178930 \\
4.706490 \\
4.706490 \\
6.236730\end{array}$ & $\begin{array}{l}0.232680 \\
0.213191 \\
0.239184 \\
0.218641 \\
0.365906 \\
0.365906 \\
0.232119\end{array}$ & $\begin{array}{l}2.236480 \\
1.345070 \\
1.231280 \\
0.776088 \\
3.294060 \\
5.869920 \\
5.450760\end{array}$ \\
\hline $0.198^{-}$ & $\begin{array}{l}1 \\
2 \\
3 \\
4 \\
5 \\
6 \\
7\end{array}$ & $\begin{array}{l}3.056240 \\
3.249760 \\
3.671280 \\
4.072330 \\
4.564490 \\
4.803120 \\
6.841540\end{array}$ & $\begin{array}{l}0.084201 \\
0.280080 \\
0.330901 \\
0.365187 \\
0.333281 \\
0.356770 \\
0.017379\end{array}$ & $\begin{array}{l}0.433911 \\
3.620430 \\
1.736750 \\
1.710470 \\
3.447300 \\
5.812080 \\
5.475890\end{array}$ & 0.700 & $\begin{array}{l}1 \\
2 \\
3 \\
4 \\
5 \\
6 \\
7\end{array}$ & $\begin{array}{l}3.466160 \\
3.695340 \\
3.951350 \\
4.197670 \\
4.703400 \\
4.703400 \\
6.057890\end{array}$ & $\begin{array}{l}0.231875 \\
0.199884 \\
0.199637 \\
0.195274 \\
0.369344 \\
0.369344 \\
0.221071\end{array}$ & $\begin{array}{l}1.992050 \\
1.357960 \\
1.008280 \\
0.740145 \\
3.463190 \\
5.869920 \\
5.047800\end{array}$ \\
\hline 0.315 & $\begin{array}{l}1 \\
2 \\
3 \\
4 \\
5 \\
6 \\
7\end{array}$ & $\begin{array}{l}3.121470 \\
3.315060 \\
3.725290 \\
4.110960 \\
4.649450 \\
4.765640 \\
6.837320\end{array}$ & $\begin{array}{l}0.105011 \\
0.284593 \\
0.322190 \\
0.356399 \\
0.337211 \\
0.409381 \\
0.025715\end{array}$ & $\begin{array}{l}0.519449 \\
3.451430 \\
1.568600 \\
1.524940 \\
3.564840 \\
5.869920 \\
5.330970\end{array}$ & 0.804 & $\begin{array}{l}1 \\
2 \\
3 \\
4 \\
5 \\
6 \\
7\end{array}$ & $\begin{array}{l}3.579240 \\
3.785860 \\
3.997220 \\
4.212350 \\
4.708380 \\
4.708380 \\
6.082540\end{array}$ & $\begin{array}{l}0.229747 \\
0.180598 \\
0.176558 \\
0.173953 \\
0.390873 \\
0.390873 \\
0.156314\end{array}$ & $\begin{array}{l}1.653770 \\
1.254100 \\
0.909090 \\
0.625824 \\
3.898090 \\
5.869920 \\
4.873670\end{array}$ \\
\hline 0.419 & $\begin{array}{l}1 \\
2 \\
3 \\
4 \\
5 \\
6\end{array}$ & $\begin{array}{l}3.218530 \\
3.443350 \\
3.788520 \\
4.129240 \\
4.705230 \\
4.705230\end{array}$ & $\begin{array}{l}0.186985 \\
0.244430 \\
0.290906 \\
0.282572 \\
0.377187 \\
0.377187\end{array}$ & $\begin{array}{l}1.516500 \\
2.126240 \\
1.546290 \\
1.081240 \\
3.532650 \\
5.869920\end{array}$ & & & & & \\
\hline
\end{tabular}

$$
E_{3}=a_{0}+a_{1} x+a_{3} x^{2}+a_{4} x^{3} .
$$

Rather than fitting to the coefficients in Table II (a procedure which would produce significant compromises in the accuracy of the estimated dielectric constants), we fitted to all nine $(x=0-0.804)$ data sets simultaneously to yield the best cubic coefficients to minimize the errors in the dielectric response over the entire range. The resulting coefficients are given in Table III. The resulting functions yield somewhat different values than those given in Table II, since each set of values in this table are the result of best fits of the oscillator coefficients to a single $\mathrm{Al}$ mole fraction; however, these functions will yield reasonably high quality fits to the dielectric response data. The quality of fit of the cubic parametrization is virtually as good as that of Table 


Al Fraction $\quad \alpha \quad E_{\alpha}(\mathrm{eV}) \quad \Gamma_{\alpha}(\mathrm{eV}) \quad\left|A_{\alpha}\right| \phi_{\alpha}$ (radians)

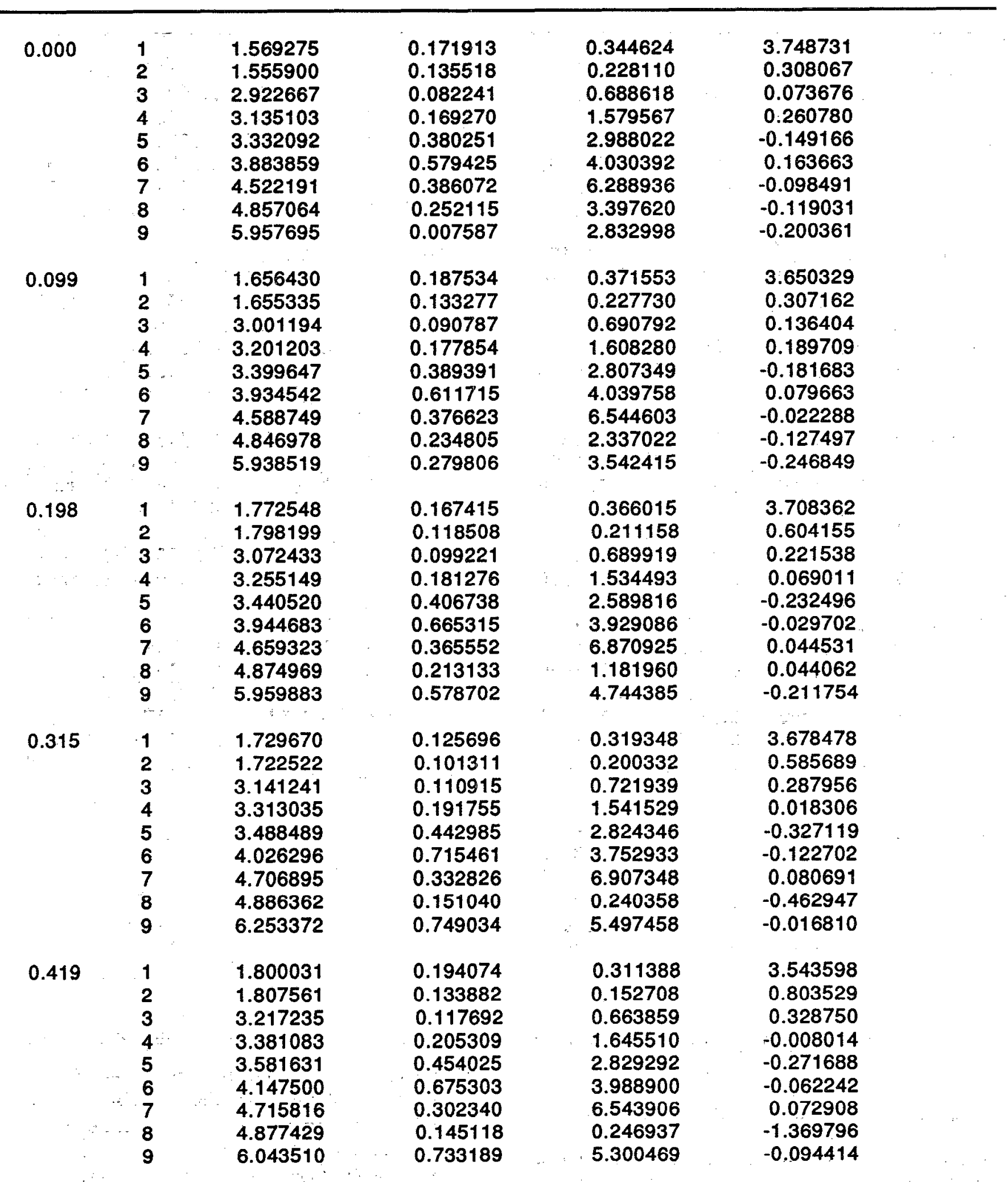




$\begin{array}{llllll}\text { Al Fraction } & \alpha & E_{\alpha}(\theta \mathrm{V}) & \Gamma_{\alpha}(\theta \mathrm{V}) & \mid \mathrm{A} \alpha & \phi_{\alpha} \text { (radians) }\end{array}$

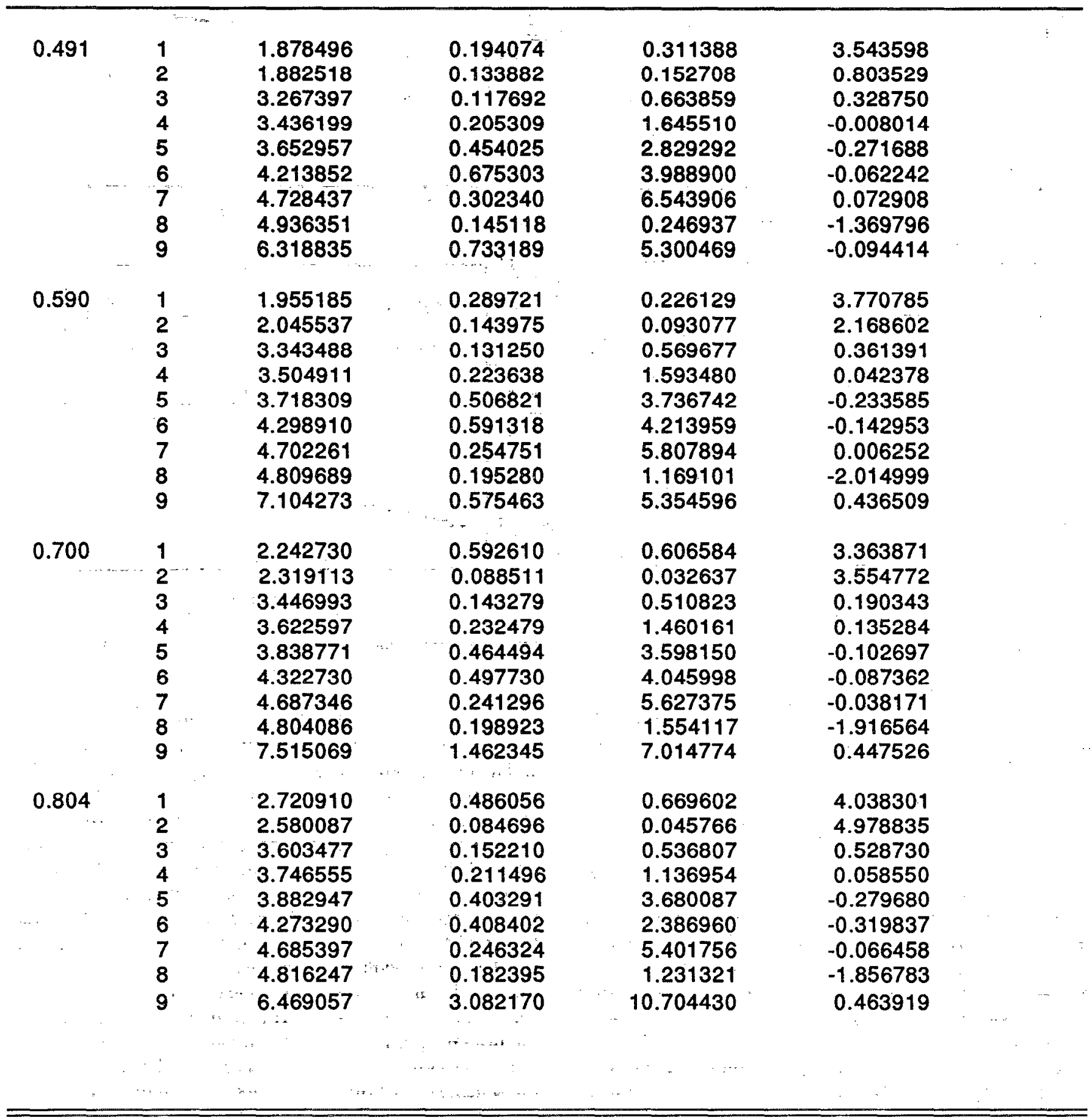

II, except at $x=0.198$ and $x=0.315$, where the cubic model does not reproduce the data around $E_{0}$ as accurately. For brevity, in the tests of the models which are discussed below, we report only the results of the linear interpolation scheme using the values from Table II; however, in most cases, the differences in derived quantities (thicknesses and mole fractions) between the two interpolation schemes are negligible.

\section{SIMULATION AND EXPERIMENT}

We have examined the quality of the modified harmonic oscillator model using both simulations of spectroscopic ellipsometry measurements ùsing Aspnes' data, and by analysis of experimental data using MBE and MOCVD grown structures. Simulated and experimental data were analyzed by a Levenberg-Marquardt minimization rou- 


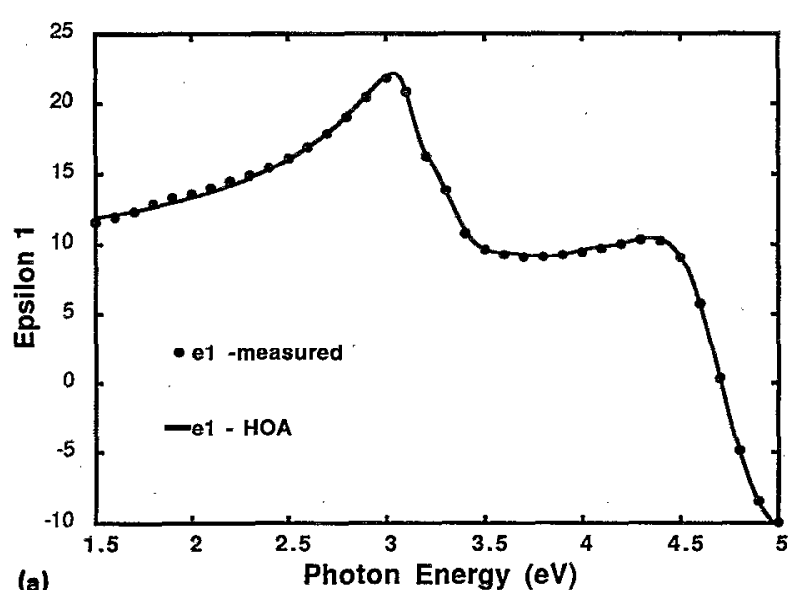

(a)

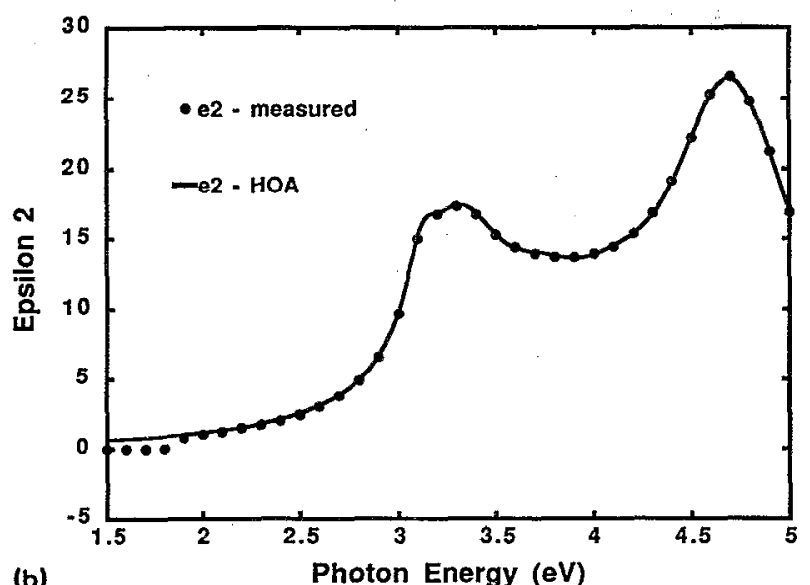

(b)

FIG. 1. Seven oscillator fit to (a) $\epsilon_{1}$ and (b) $\epsilon_{2}$ for $\mathrm{Al}_{0.315} \mathrm{Ga}_{0.685}$ As.

tine ${ }^{10}$ based on comparison of the data with the model for the structure using the error function $f$ :

$$
\begin{aligned}
f= & \frac{1}{N}\left(\sum_{i=1}^{N}\left[\tan (\psi)_{i}^{m}-\tan (\psi)_{i}^{c}\right]^{2}\right. \\
& \left.+\left[\cos (\Delta)_{i}^{m}-\cos (\Delta)_{i}^{c}\right]^{2}\right),
\end{aligned}
$$

where the superscripts $m$ and $c$ refer to measured and calculated, respectively, and the summation is over the $N$ different photon energies. The error is measured in terms of the standard unbiased estimator $\sigma$ and the $95 \%$ confidence limits for each fitted parameter were calculated using standard techniques. ${ }^{9}$

First, we examined the model by simulating the spectroscopic ellipsometry data that would result from a measurement of the structure shown in Fig. 3. This structure was chosen because it is typical of many MODFET-type device structures. We assumed a measurement angle of incidence of $75^{\circ}$, since this would provide significant sensitivity to the buried AlGaAs layer. Regression analysis was performed with a wide variety of starting points for thickness and $\mathrm{Al}$ mole fraction, and all fits converged to the same results (within insignificant numerical errors). The numeric results of the regression analysis using both the seven oscillator scheme and our nine oscillator scheme are
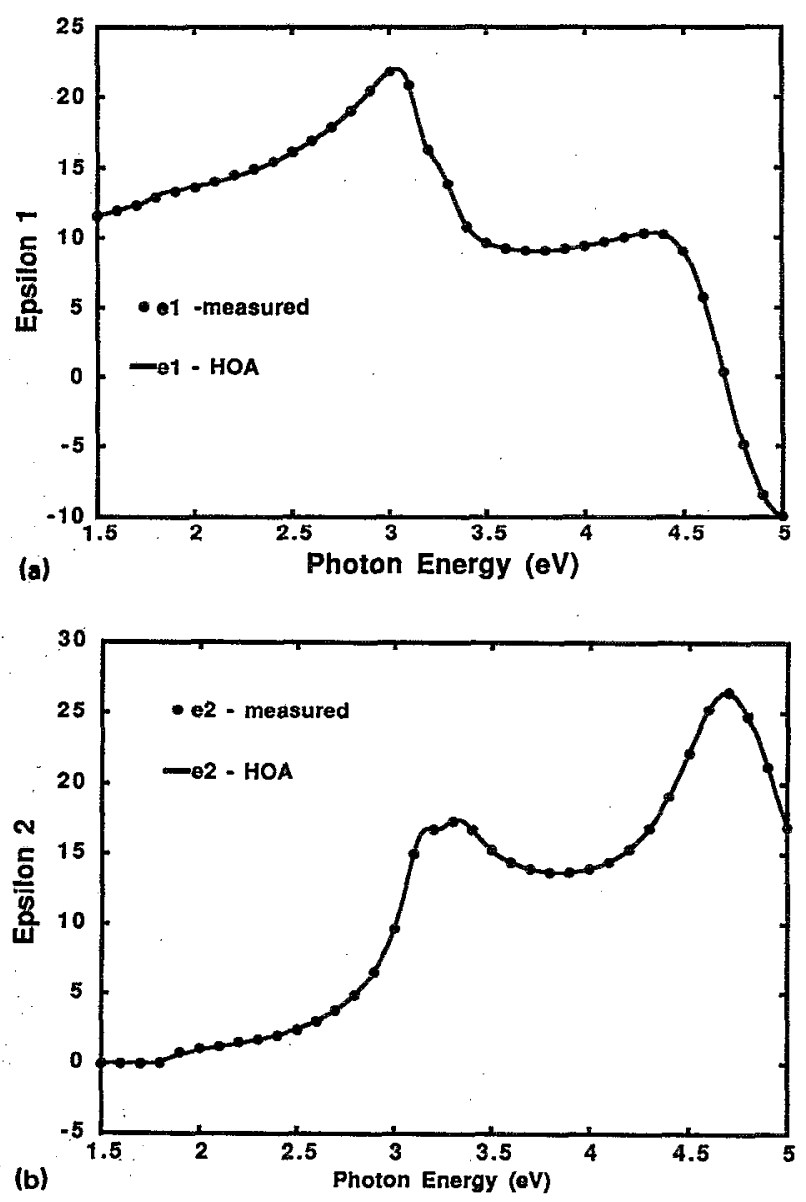

FIG. 2. Nine oscillator fit to (a) $\epsilon_{1}$ and (b) $\epsilon_{2}$ for $\mathrm{Al}_{0.315} \mathrm{Ga}_{0.685} \mathrm{As}$.

summarized in Table IV. The regression fit versus simulation for the nine oscillator approximation is shown in Fig. 4. The fit is excellent everywhere except around $\sim 1.9 \mathrm{eV}$, where the harmonic oscillator-based regression fit shows a somewhat higher $\cos \Delta$ value. This is due to slight deviations in $\epsilon_{1}$ and $\epsilon_{2}$ in the harmonic oscillator model versus Aspnes' data around $E_{0}$. As shown in Table IV, the fit using the seven oscillator scheme has a much higher $\sigma$, and has significant errors in the GaAs and AlGaAs thicknesses. For brevity we have not shown the plots of $\tan \psi$ and $\cos \Delta$ for the seven oscillator fit, however, the quality of the fit between 1.5 and $1.9 \mathrm{eV}$ was significantly worse in this case. This problem was not surprising, since in this structure, the primary sensitivity of the simulation to AlGaAs thickness is in the lower-energy region, where the seven oscillator scheme is not applicable. These simulations illustrate that for realistic device structures, the nine oscillator scheme can be used for high accuracy thickness and composition extractions from spectroscopic ellipsometry measurements.

Two very simple experimental tests were performed to check the behavior of our model. First, a simple GaAs substrate was measured following cleaning using a 50\% $\mathrm{NH}_{4} \mathrm{OH}-\mathrm{H}_{2} \mathrm{O}$ solution. The sample was measured at an angle of incidence of $70^{\circ}$ in room air. The data were analyzed assuming $\mathrm{GaAs}$ oxide on an $\mathrm{Al}_{x} \mathrm{Ga}_{1}-x$ As substrate. 


\begin{tabular}{|c|c|c|c|c|c|c|c|c|c|}
\hline Paramete & $a_{0}$ & $a_{1}$ & $a_{2}$ & $a_{3}$ & Paramete & $a_{0}$ & $a_{1}$ & $a_{2}$ & $a_{3}$ \\
\hline$E_{1}$ & 1.39060 & 2.09260 & -1.60624 & 1.39457 & $E_{6}$ & 3.89007 & 1.34836 & -0.46618 & -0.47756 \\
\hline$\Gamma_{1}$ & 0.13786 & 0.13697 & 0.47712 & 0.09325 & $\Gamma_{6}$ & 0.79424 & 0.11166 & -0.20242 & -0.13491 \\
\hline$A_{1}$ & 0.36529 & 0.08038 & -2.23761 & 4.07322 & $A_{6}$ & 5.43232 & -0.37707 & 8.27471 & -3.76629 \\
\hline$\phi_{1}$ & 2.53491 & 1.37492 & 2.81830 & -2.88902 & $\phi_{6}$ & 0.33913 & -0.31718 & 1.03742 & -0.19826 \\
\hline$E_{2}$ & 1.40068 & 1.99832 & -1.90326 & 1.53105 & $E_{7}$ & 4.55820 & 0.43356 & -0.25474 & -0.08739 \\
\hline$\Gamma_{2}$ & 0.11079 & -0.17490 & 1.26335 & -1.36715 & $\Gamma_{7}$ & 0.33443 & -0.19031 & 0.01285 & -0.06472 \\
\hline$A_{2}$ & 0.26138 & -0.36973 & 0.40233 & -0.34884 & $\mathrm{~A}_{7}$ & 5.21290 & -5.39337 & 1.05408 & -1.05662 \\
\hline$\phi_{2}$ & -0.88229 & 1.14488 & -2.90855 & 0.72062 & $\phi_{7}$ & 0.30805 & 0.92272 & -0.71698 & 1.17884 \\
\hline$E_{3}$ & 2.92232 & 0.82560 & -0.59311 & 0.83462 & $E_{8}$ & 4.83763 & -0.29928 & 0.40196 & -0.27251 \\
\hline$\Gamma_{3}$ & 0.08267 & 0.07536 & 0.00580 & 0.02843 & $\Gamma_{8}$ & 0.27022 & 0.19000 & -0.18688 & 0.03856 \\
\hline$A_{3}$ & 0.70159 & -0.47910 & 1.68160 & -0.85775 & $A_{8}$ & 4.85697 & -1.38754 & 3.82283 & 0.52175 \\
\hline$\phi_{3}$ & 0.04704 & 0.42993 & 1.61326 & 0.42177 & $\phi_{8}$ & -0.24016 & -1.25824 & 1.17243 & -0.32264 \\
\hline$E_{4}$ & 3.14001 & 0.65211 & -0.41695 & 0.55666 & $E_{9}$ & 5.70697 & 1.82111 & -2.62439 & 2.34028 \\
\hline$\Gamma_{4}$ & 0.16014 & 0.11849 & -0.16197 & 0.13568 & $\Gamma_{9}$ & 0.37446 & 0.54285 & -6.79344 & 9.63119 \\
\hline$A_{4}$ & 1.36908 & -0.73488 & 3.34016 & -0.96149 & $A_{9}$ & 3.90983 & 0.67149 & -10.27836 & 16.38186 \\
\hline$\phi_{4}$ & 0.33154 & -0.77594 & 0.91058 & -1.06396 & $\phi_{9}$ & -0.70131 & 2.01631 & -1.32069 & -0.21253 \\
\hline$E_{5}$ & 3.25876 & 0.46874 & 0.17375 & -0.04572 & & & & & \\
\hline$\Gamma_{5}$ & 0.38313 & 0.18661 & -0.23121 & -0.19555 & & & & & \\
\hline$A_{5}$ & 2.87457 & 1.49872 & -3.40598 & -0.21577 & & & & & \\
\hline$\phi_{5}$ & -0.49340 & 0.04123 & -1.76056 & 0.14619 & & & & & \\
\hline
\end{tabular}

Regression analysis yielded: $\sigma=5.9 \times 10^{-3}$, an oxide thickness of $7.0 \pm 0.5 \AA$, and $x=0.016 \pm 0.006$. This small compositional error could be the result of scveral factors including instrument inaccuracies, deviations of the native oxide dielectric constants from the values of anodic oxides, or some other residual film on the surface.

A second simple experimental test was performed on an MOCVD grown $\mathrm{Al}_{x} \mathrm{Ga}_{1}{ }_{x}$ As on GaAs sample. The AlGaAs was approximately $2 \mu \mathrm{m}$ thick with $x \approx 0.4$. The AlGaAs contained no intentionally introduced "dopants and was grown on a semi-insulating GaAs substrate. Several measurements were taken on the samplc as grown and following the use of $\mathrm{Br}$-methanol and $\mathrm{NH}_{4} \mathrm{OH}-\mathrm{H}_{2} \mathrm{O}$ stripping solutions as suggested by Aspnes. ${ }^{1}$ Our spectroscopic ellipsometry system is currently not equipped to do these steps in situ; therefore, the measurements were done immediately following chemical treatments. The measurements were performed at room temperature. The data were analyzed assuming a single layer model (native oxide on an AlGaAs substrate). Some interference effects due to the finite AlGaAs thickness were noted, but these were much smaller than expected from a two-film model. This could result from compositional gradients in the AlGaAs, or a rough or chemically mixed interface with the GaAs substrate. The regression analysis was performed assuming that the surface oxide had the optical constants of GaAs oxide. We attempted to obtain the $E_{1}$ critical point energy by converting the measured data into pseudodielectric constants using a single layer (native oxide) on infinite substrate model and then fitting 3D-M1-type lineshape functions to $\partial^{3}\left(E^{2} \epsilon_{1}\right) / \partial E^{3}$ (the joint density of states derivative). The Al mole fraction was obtained from the $E_{1}$ critical point energy using formula (2) of Aspnes et al..$^{5}$ The best agreement between these two measurements was obtained on an unetched sample at an angle of $75^{\circ}$. Regression analysis yielded an unbiased estimator of $4.05 \times 10^{-2}$ 


\begin{tabular}{|l|}
\hline Layer 1: $10 \AA$ GaAs Oxide \\
\hline Layer 2: $500 \AA$ GaAs \\
\hline Layer 3: $400 \AA$ AlGaAs $\times=0.315$ \\
\hline GaAs Substrate \\
\hline
\end{tabular}

FIG. 3. MODFET-type structure used to create ideal data for testing harmonic oscillator schemcs.

with $16.7 \pm 1.0 \AA$ A of oxide and $x=0.421 \pm 0.007$. The $E_{1}$ energy was estimated to be $3.195 \mathrm{eV}$, corresponding to $x=0.419$. Most of the error in the HOA regression fit was due to disagreements around the sharp features in $\epsilon$ at $E_{1}$ and $E_{1}+\Delta_{1}$. This disagreement appears similar to the problems encountered by Aspnes in measurement of high Al-concentration samples; therefore, we believe it to be the result of oxidation due to our measurements being done in air. Larger differences between the HOA regression fit and the $E_{1}$ point estimates for $x$ were found on samples subjected to the etching steps. For instance, following an etch, a sample yielded $x$ values of $0.476 \pm 0.014$ using the HOA regression fit and 0.422 from the $E_{1}$ point. We believe that these problems were caused by oxidation of our samples ion room air following the chemical etching. Also, it appears from these experiments that the $E_{1}$ critical point position provides a measurement of $x$ that is less sensitive to surface preparation than the value obtained from the nondifferentiated regression method.

A double-crystal $x$-ray rocking curve measurement of a sample from a wafer in the same MOCVD run produced a pair of well-defined, narrow peaks separated by 160.4 arc$\mathrm{sec}$, yielding an $\mathrm{Al}$ fraction of approximately 0.449 . This is reasonably close to the value obtained from ellipsometric analysis. Also; spectroscopic ellipsometry measurements of several samples from this lot indicated possible Al compositions from about 0.42 to 0.5 ; therefore, agreement may be better than the $\sim 0.03$ error indicated by the above. The results on this sample indicate that our interpolation technique will yield approximately the correct Al mole fraction, but more strongly illustrate the problem of obtaining

TABLE IV. Comparison of the results of regression analysis of simulated data for the structure shown in Fig. 3.

\begin{tabular}{ccrc}
\hline \hline Layer & Simulation & Nine oscillator model & Seven oscillator model \\
\hline 1 & $10 \AA$ & $9.4 \pm 0.3 \AA$ & $12.3 \pm 0.6 \AA$ \\
2 & $500 \AA$ & $496.0 \pm 4.2 \AA$ & $588.9 \pm 11.6 \AA$ \\
3 & $400 \AA$ & $398.8 \pm 10.3 \AA$ & $288.7 \pm 23.1 \AA$ \\
3 & $x=0.315$ & $x=0.315 \pm 0.004$ & $x=0.309 \pm 0.033$ \\
& & $\sigma=8.86 \times 10^{-3}$ & $\sigma=1.94 \times 10^{-2}$ \\
\hline
\end{tabular}
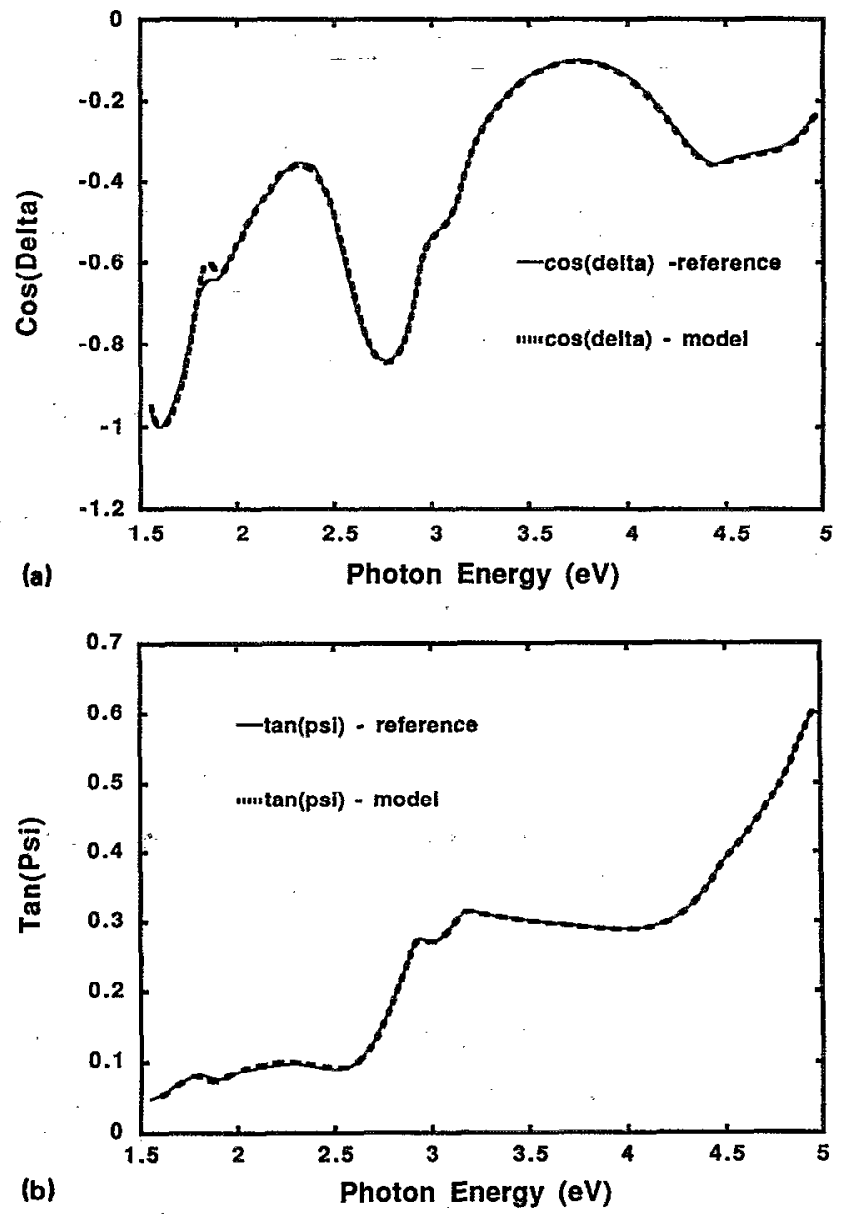

FIG. 4. Comparison of a simulated MODFET structure from Fig. 3 using reference data and regression analysis of the reference data using the nine oscillator model for (a) $\cos \Delta$ and (b) $\tan \psi$.

good ellipsometric measurements on samples with high $\mathrm{Al}$ content.

A more complex sample was evaluated to further test the applicability of our model to multilayer sample analysis. The structure was MBE grown using conventional solid source techniques with a targeted structure shown in Fig. 5. The sample was measured at an angle of incidence

\begin{tabular}{|l|}
\hline Layer 1: native oxide \\
\hline Layer 2: $2000 \AA$ GaAs \\
\hline Layer 3: $500 \AA$ AlGaAs $x=0.25$ \\
\hline Layer 3: $1000 \AA$ AlGaAs $x=0.65$ \\
\hline GaAs Substrate \\
\hline
\end{tabular}

FIG. 5. Targeted values for an MBE-grown GaAs/AlGaAs structure analyzed using spectroscopic ellipsometry and the nine oscillator scheme. 
TABLE V. Comparison of the results of regression analysis and targeted values for the structure shown in Fig. 5. The measured data used regression analysis employing the nine oscillator model.

\begin{tabular}{ccc}
\hline \hline Layer & Target & Measured \\
\hline 1 & $\ldots$ & $18.3 \pm 0.6 \AA$ \\
2 & $2000 \AA$ & $2015.8 \pm 22.3 \AA$ \\
3 & $500 \AA$ & $479.5 \pm 11.2 \AA$ \\
3 & $x=0.25$ & $x=0.199 \pm 0.009$ \\
4 & $1000 \AA$ & $861.0 \pm 18.5 \AA$ \\
4 & $x=0.65$ & $x=0.675 \pm 0.023$ \\
& & $\sigma=1.50 \times 10^{-2}$ \\
\hline
\end{tabular}

of $75^{\circ}$ to improve the sensitivity to the buried AlGaAs layers and regression analysis was performed on the resulting data over the $1.65-4.13 \mathrm{eV}$ range. The results of the analysis are given in Table $\mathrm{V}$, and comparison of the measured data and the regression fit are shown in Fig. 6. Critical point analysis could not be performed for the $\mathrm{Al}$ mole fractions due to absorption in the top GaAs layer. Rocking curve measurements did not yield clear peaks for the epitaxial films due to their small thicknesses, and, therefore, verification of the compositions proved to be impractical. We are currently attempting to use transmission electron microscopy (TEM) to directly confirm these thicknesses; however, Woollam et al., ${ }^{4}$ have previously demonstrated
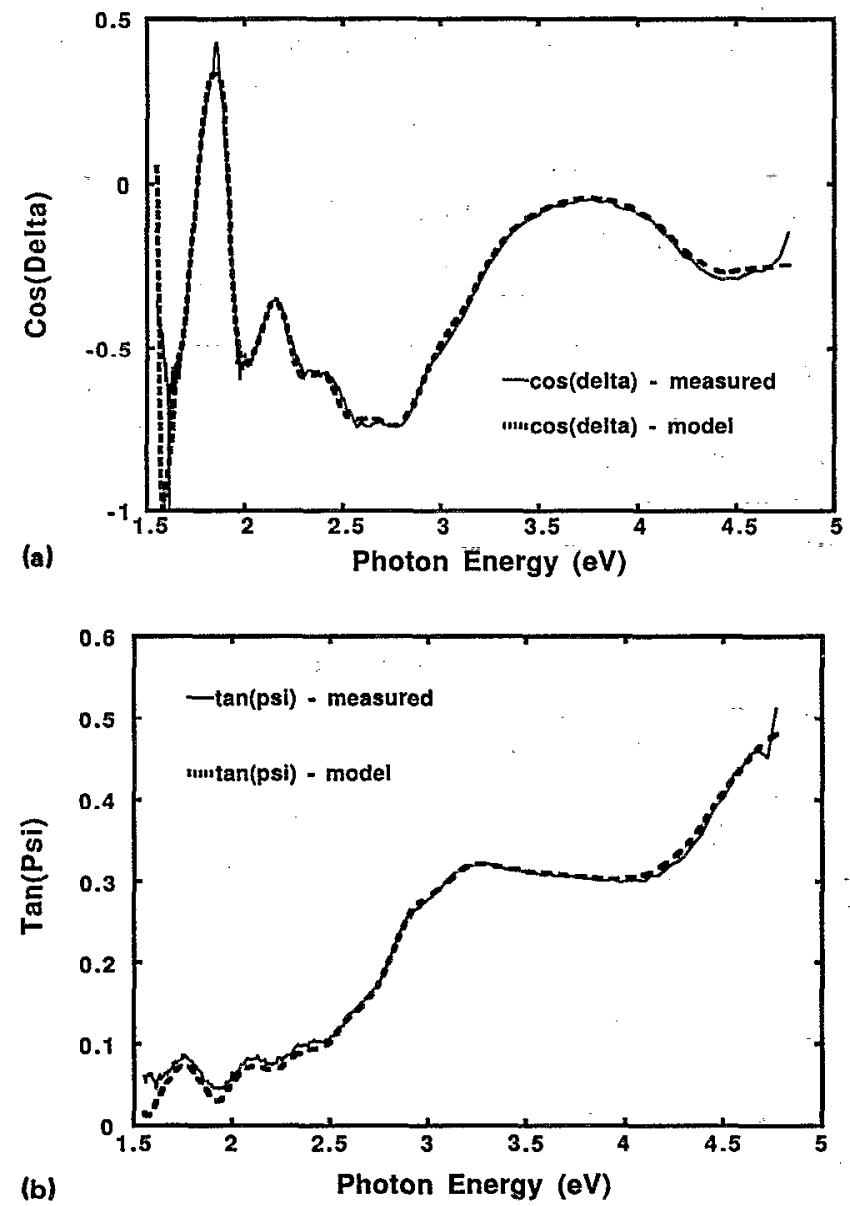

(b)

FIG, 6. Comparison of measured data and regression analysis using the nine oscillator model for the $\mathrm{GaAs} / \mathrm{Al}_{x 1} \mathrm{Ga}_{1-x 1} \mathrm{As} / \mathrm{Al}_{x 2} \mathrm{Ga}_{1-x 2} \mathrm{As} / \mathrm{GaAs}$ structure of Fig. 5 for (a) $\cos \Delta$ and (b) $\tan \psi$. that spectroscopic ellipsometry is capable of yielding measurement accuracies comparable to those of TEM in this material system. The quality of fit shown in Fig. 6 could not have been obtained if there were large errors in the interpolated dielectric constants. Therefore, we feel that this experiment, while not directly supported by an independent measurement, provides good evidence for the usefulness of this approximation scheme.

\section{DISCUSSION}

We have demonstrated a comparatively simple scheme for analytically approximating the dielectric constants of the $\mathrm{Al}_{x} \mathrm{Ga}_{1-x} \mathrm{As}$ family, and have demonstrated its usefulness using computational and experimental tests. While other approaches can certainly be found, this approach offers relatively high numerical efficiency and sufficient accuracy for numerically intensive modeling problems such as spectroscopic ellipsometry data analysis. These approximations should also prove useful for other optical problems involving these materials, such as solar cell design.

This approach should be applicable to other well characterized ternary compound systems and for quaternary systems where only one compositional variable can be considered independent (such as latticed-matched $\mathrm{In}_{x} \mathrm{Ga}_{1-x} \mathrm{As}_{y} \mathrm{P}_{1-y}$ on InP). We are currently working on application of this technique to strained and latticed matched $\mathrm{In}_{x} \mathrm{Ga}_{1-x} \mathrm{As}$ on $\mathrm{InP}$ and latticed-matched $\operatorname{In}_{x} \mathrm{Ga}_{1-x} \mathrm{As}_{y} \mathrm{P}_{1-y}$ on InP.

\section{ACKNOWLEDGMENTS}

The author would like to acknowledge the HewlettPackard Corporation for the donation of a computer work station on which the computation for this work was performed, Dr. Jack East for providing the MBE sample, and Dr. Michael Elta and Dr. Andrew Robinson for helpful discussions. This work was supported by the U. S. Army Research Office under the contract No. DAAL03-87-K0007.

${ }^{1}$ M. Erman, J. B. Theeten, N. Vodjdani, and Y. Demay, J. Vac. Sci. Technol. B 1, 328 (1983).

${ }^{2}$ S. A. Alterovitż, P. G. Synder, K. G. Merkel, J. A. Woollam, D. C. Radulescu, and L. F. Eastman, J. Appl. Phys. 63, 5081 (1988).

${ }^{3}$ K. G. Merkel, P. G. Synder, J. A. Woollam, S. A. Alterovitz, and A. K. Rai, Jpn. Appl. Phys. 28, 1118 (1989).

${ }^{4}$ J. A. Woollam, P. G. Snyder, A. W. McCormick, A. K. Rai, D. Ingram, and P. P. Pronko, J. Appl. Phys. 62, 4867 (1987).

${ }^{5}$ D. E. Aspnes, S. M. Kelso, R. A. Logan, and R. Bhat, J. Appl. Phys. 60, 754 (1986).

${ }^{6}$ M. Erman, J. B. Theeten, P. Chambon, S. M. Kelso, and D. E. Aspnes, J. Appl. Phys. 56, 2664 (1984).

${ }^{7}$ S. Adachi, Phys. Rev. B 38, 12345 (1988).

${ }^{8}$ D. W. Jenkins, J. Appl. Phys. 68, 1848 (1990).

${ }^{9}$ M. Erman and J. B. Theeten, Surf. Sci. 135, 353 (1983).

${ }^{10}$ W. H. Press, B. P. Flannery, S. A. Teukolsky, and W. T. Vetterling, Numerical Recipes: The Art of Scientific Computing (Cambridge University Press, Cambridge, England), 523ff.

${ }^{11}$ Copies of these tables can be obtained on HP, Macintosh, IBM PC, and some other computer readable magnetic by contacting the author. Copies may also be received via electronic mail be sending requires to fred terry@um.cc.umich.edu. 